\title{
"Scuola Sicura": a school screening program to prevent the spread of COVID-19 among students in Piedmont, Italy
}

\section{Elena Farina}

Epidemiology Unit - ASL TO3, Piedmont Region

Ilenia Eboli

Epidemiology Unit - ASL TO3, Piedmont Region

Teresa Spadea ( $\nabla$ teresa.spadea@epi.piemonte.it)

Epidemiology Unit - ASL TO3, Piedmont Region

\section{Carlo Saugo}

School of Hygiene and Preventive Medicine, University of Turin

\section{Lorenzo Richiardi}

Department of Medical Sciences, University of Turin

\section{Milena Maule}

Department of Medical Sciences, University of Turin

\section{Pietro Presti}

Piedmont Region

\section{Antonella Bena}

Epidemiology Unit - ASL TO3, Piedmont Region

\section{Research Article}

Keywords: Covid-19, Screening, School

Posted Date: October 4th, 2021

DOI: https://doi.org/10.21203/rs.3.rs-864946/v2

License: (1) (1) This work is licensed under a Creative Commons Attribution 4.0 International License. Read Full License 
"Scuola Sicura": a school screening program to prevent the spread of COVID-19 among students in Piedmont, Italy

Elena Farina, ${ }^{1}$ Ilenia Eboli, ${ }^{1}$ Teresa Spadea, ${ }^{1}$ Carlo Saugo, ${ }^{2}$ Lorenzo Richiardi, ${ }^{3}$ Milena Maule, ${ }^{3}$ Pietro Presti, ${ }^{4}$ Antonella Bena ${ }^{1,5}$

${ }^{1}$ Epidemiology Unit - ASL TO3, Piedmont Region

${ }^{2}$ School of Hygiene and Preventive Medicine, University of Turin

${ }^{3}$ Department of Medical Sciences, University of Turin

${ }^{4}$ Piedmont Region

${ }^{5}$ Regional Documentation Center for Health Promotion (DoRS), Piedmont Region

Corresponding author: Teresa Spadea

Epidemiology Unit - ASL TO3

Via Sabaudia 164 - 10095 Grugliasco (Torino)

teresa.spadea@epi.piemonte.it 


\begin{abstract}
Objectives

Aims of "Scuola Sicura" (SS) were to monitor the rate of COVID-19 and to contain its spread within the school population through early case isolation. We report the initial process and outcome evaluation results.

\section{Design}

Descriptive study of an experimental screening testing program in children in Piedmont in the period January-March 2021. We used data from the COVID-19 platform and the Local Health Units, the archives of birth certificates (CedAP) and hospital discharge files (SDO).
\end{abstract}

\title{
Setting and participants
}

The screening program targeted second and third grade students in first level secondary schools. Participants were subdivided into four groups; one group each week underwent screening, yielding one test per student per month.

\section{Main outcome measures}

We calculated:

1. number of positive cases detected vs. total number of students tested in the SS program;

2. number of positive cases detected outside the SS program vs. total number of students in the target population.

We detected the number of quarantines due to SS and no-SS case identification. To investigate the spread of COVID-19 in households, the mother-child pairs were identified through record linkage between the CedAP and SDO archives, and positive mothers were identified.

\section{Results}

Sixty-nine percent of schools and $19.5 \%$ of the students participated in the program. SS detected 114 positives cases for SARS-CoV-2. On 08.03.2021, the target classes started distance learning: 69 of the 114 positive students were identified before that date, leading to the activation of 67 quarantine measures. We were able to identify the mothers of 61 out of 69 of those students (88\%); 46 mothers had performed a swab test after the positivity of their child with a positive result in 11 cases. Asymptomatic cases identified at screening during in-class learning period accounted for $26.5 \%$ of the total number of cases occurred in the participating classes.

\section{Conclusions}

This is one of the few studies (and the first in Italy) to describe the functioning and predictive capacity of school screening testing for SARS-CoV-2 in a real-world situation. Our findings provide data-driven suggestions for government agencies when planning large-scale school screening testing programs.

Keywords: COVID-19, Screening, School 


\section{What was already known}

- Children and young people are less susceptible to contracting COVID-19 than other age segments of the population; often asymptomatic or mildly, they can still carry and spread the virus;

- though its effectiveness was not demonstrated, one of the measures many countries adopted to contain the pandemic was repeated complete or partial school closure, with consequent negative effects on psychophysical health and learning;

- screening tests for SARS-COV2 in schools have been experimented in many countries and in some Italian regions, but the cost-effectiveness of this approach is unclear.

\section{What this study adds}

- The practical application of a structured school screening program for SARS-COV2 requires coordinated school-health work and careful communication to families;

- in a period of high spread of the virus in the community, the screening identified a quarter of the cases that occurred in the participating classes;

- structured school screening provides an important contribution in identifying and isolating asymptomatic cases; jointly with contact tracing and other measures of transmission prevention, it may be a viable strategy to keep schools open when high levels of the virus are circulating in the community. 


\section{Introduction}

Children and young people are less susceptible to contracting COVID-19 than other age segments of the population. They are often asymptomatic or mildly symptomatic ${ }^{1,2}$, but can still spread the virus. Temporary complete or partial school closure was adopted by many countries as a measure to control the COVID-19 pandemic, even if the exact effectiveness if this measure is still unclear. ${ }^{3}$ Keeping schools open, however, is crucial to support children's psychophysical health, assure continuity of learning, and limit education inequalities. ${ }^{4,5}$

The core approach to contain the spread of COVID-19 in schools involves nonpharmacological measures: physical distancing, use of face masks, ventilation of indoor environments, frequent handwashing, disinfection of hard surfaces. ${ }^{6,7}$ Diagnostic testing of children with symptoms, and timely contact tracing can help keeping schools open. ${ }^{8,9}$ Many countries have carried out screening of children and staff for the early detection of infectious cases without symptoms in compulsory schools ${ }^{10}, 9,11$ and universities..$^{12}$ The cost-effectiveness of this approach in low-prevalence settings is unclear. ${ }^{13}$

Some Regional governments in Italy have implemented similar actions; some schools encouraged their students to take part in screening tests when they reopened after Christmas breaks. The Italian Ministry of health has financed a project in which pooled screening tests is experimented as an early warning strategy to identify COVID-19 outbreaks in selected schools of five regions (https://www.ccm-network.it/progetto.jsp?id=node/2030\&idP=740). In all these cases, testing was variously organized, carried out using different types of tests, in different phases of the pandemic. In December 2020, the regional government of Piedmont approved an experimental screening program entitled "Scuola Sicura" (SS) with the aim of monitoring the transmission rate of COVID-19 and trying to contain more rapidly its spread within the school population through early case isolation. The first phase of the program lasted from January to March 2021.

The objective of this paper is to describe and discuss the functioning of the proposed screening program in a real context. We analyzed the adherence to the program, described the time trends of cases identified by the screening among tested students compared to the trend outside the program, and assessed the number of asymptomatic cases identified beyond the standard procedures of contact tracing and the consequent quarantines.

\section{The program}

The screening program targeted second and third grade students in first level secondary schools throughout Piedmont. Participation was voluntary and could be withdrawn at any time. Written, informed consent was obtained from parents. Only classes in which at least $25 \%$ of the students volunteered to participate were enrolled in the screening. Within each of these, participants were subdivided into four groups; one group each week underwent screening, yielding one test per student per month. ${ }^{14}$

Screening was managed independently by the twelve Local Health Units (LHU) in Piedmont in collaboration with the schools. The school principal's offices handled communication with the families, collected informed consent, and provided the lists of participating classes and students to the LHU. Each LHU was responsible for test scheduling and dealing with positive test results. Students were accompanied by their parents to the designated testing center, where they received a molecular or antigen swab test, according to availability in each LHU. Positive cases were defined as a positive molecular swab test or a positive antigen swab test confirmed by molecular testing. In rare cases, particularly during the pick of the pandemic when the test positive predictive values were higher, some LHUs did not require confirmation by molecular testing.

When a student tested positive, the LHU evaluated the potential risk of transmission according to the Ministry of Health criteria: if the positive student had been in contact with his/her classmates in 
the 48 hours preceding the test, the entire class was quarantined for 14 days without carrying out any further test within the class. If the antigen swab test was followed by a negative molecular test, the quarantine was discontinued.

The data presented here were collected between January and March, when the infection rate in Piedmont was particularly high. Screening was performed for 2 months; some LHUs began screening before others, so that the entire study period is approximately 3 months.

\section{Material and methods}

\section{Data Sources}

Data from the COVID-19 platform

The data for the present study primarily came from the Piedmont COVID-19 Platform, a management software that traces and monitors the data of COVID-19 patients, created by the Consortium for the Piedmont information Systems. Integrated with regional healthcare databases, the platform allows the follow-up of all patients accessing health services for a SARS-CoV-2 test. For the SS program, an application was designed specifically for transmitting the data from LHUs to the regional database, allowing record linkage with the main tables.

The platform includes data on all molecular and antigen swab test results, including those from private laboratories, pharmacies, doctors' offices, nursing homes, LHUs, and hospitals (preadmission screening, primary care providers, etc.).

The following variables were used: type of test, result of the test (positive, negative, indeterminate), result date, and indication for the test (e.g., symptomatic case, asymptomatic but close contact with a case, confirmed negativity after a positive test result, SS screening, etc.).

\section{Local Health Unit data}

LHU data were used firstly to describe participation in the program. Each LHU produced a summary file containing the total number of students for each class in each school, the number of students who adhered to the screening program, and the number of students who had actually undergone a swab test. Moreover, since the regional platform did not enable classes identification, we used LHU data to perform the within-class comparison of positive cases detected through the screening program with those detected through the standard procedures of testing of symptomatic cases and their contacts. To this end, we collected data on the type of swab (rapid antigenic or molecular), date of positive tests, the reason why the swab was performed (screening or other), and the dates quarantine started and ended, for all the positive cases found in each class. By comparing these data with the data of the COVID-19 platform, we were able to obtain, by difference, the number of cases identified through the standard testing and contact tracing procedures among students in the classes that were not part of the screening.

\section{Data on hospital discharge and birth assistance}

In order to investigate the spread of COVID-19 in households, mother-child pairs were identified using data from the archives of the birth assistance certificates (CedAP). We extracted data on births in Piedmont between 2006 and 2009 (birth cohorts of the SS program), including out-of-region deliveries of resident women. The CedAP includes the code number of the hospital where the child was born and the medical chart number of the mother, which can be linked to the same codes included in the hospital discharge records, where anonymous identification numbers for both the newborn and his/her mother can be retrieved and the mother-child pair reconstructed. Through the anonymous identification numbers, we then linked these pairs with the COVID-19 platform to 
determine whether a swab test was performed within the SS program and its result. CeDAP records also include information on maternal educational level, which was categorized as low=none, primary/middle school; medium=high school, high=at least university degree.

\section{Statistical analysis}

The total values and percentage distribution of initial adherence to the program, actual participation, and positive swab test results were calculated for the entire Region and stratified by LHU in order to assess geographical variability in the implementation of the program.

For monitoring purposes, we compared SS results with the general surveillance statistics. Data from all the rapid antigen and molecular swab tests dated between 04 January 2021 and 28 March 2021 of the 2007-2008 birth cohorts were selected from the COVID-19 platform. Only swab tests with a definite positive or negative result belonging to students residing in Piedmont were included in the analysis.

A case was defined as positive if it had:

1. a positive molecular swab test; or

2. a positive antigen swab test confirmed by molecular testing within 7 days; or

3. a positive antigen swab test followed by a positive healing control molecular test performed between 14 and 21 days after the antigen test.

As mentioned above, a few residual cases were defined as positive even though the positive antigen swab test was not confirmed by the molecular test.

The index date was the day of the molecular test in the first instance and the date of the antigen test in the other three instances. For all positive cases, all swab tests performed for whatever reason within 21 days from the index date (maximum duration of quarantine) were eliminated, as were control swab tests even after 21 days. For positive antigen swab tests not confirmed by a subsequent molecular test, all other swab tests performed during the following 7 days were eliminated.

The swab test results were then classified as whether they were performed within the SS program or not, and stratified by week. For each week, each student was counted only once even if more than one swab test had been performed. It follows that the weekly number of persons tested refers to the students who underwent at least one swab test for that week. We calculated the following indices with their $95 \%$ confidence interval, as well as their weekly trends:

1. number of positive cases detected vs. total number of students tested in the SS program;

2. number of positive cases detected outside the SS program vs. total number of students enrolled in the 2 nd and 3rd grades of first-level secondary schools in Piedmont.

To detect turning points in the trends we used the joint point regression model (National Cancer Institute ver. 4.9.0.0) (https://surveillance.cancer.gov/jointpoint).

In order to estimate the SS preventive capacity, we used LHU data to compare the frequency of positive students and quarantines between classes participating and not participating in the SS program. Moreover, within each class, positive cases found by screening and positive cases detected by the standard testing and contact-tracing procedures were identified.

Finally, to investigate the potential spread of COVID-19 infection from children to their mothers, we first identified students found positive at the screening; we then searched the COVID-19 platform for data on mother' swab tests performed within 21 days after the child was found positive. Positive cases among the mothers were defined as previously described for the students.

On 8 March 2021, the Ministry of Health issued the decision to start remote learning nationally for first-level secondary school grades 2 and 3 . Therefore, the estimate of the SS preventive capacity 
was restricted to the period from 4 January 2021 to 7 March 2021. Time trend analysis was performed for data collected up to 28 March 2021.

The use of personal data was performed in compliance with data privacy regulations. All information collected and recorded on electronic devices was pseudonymized.

\section{Results}

\section{Adherence to SS}

A total of 428 schools (69\% of first-level secondary schools in Piedmont), and just over half (50.4\%) of the total number of school classes participated in the program (Table 1). It follows that 41,501 students (52.7\% of the students enrolled in the 2 nd and 3rd grades) attended classes covered by the SS, and thus potentially benefitted from screening, and 37,188 students attended classes in which the screening was not performed.

At the beginning of the program, 19,413 students agreed to participate $(24.7 \%$ of the target population); however, not all of them ultimately participated: some withdrew their consent, and others did not show up for the test. A total of 15,318 students (19.5\% of the target) underwent at least one swab test; $51.6 \%$ were male and $96 \%$ were born in 2007 or 2008.

Table 1 - Schools, classes and students participating in the "Scuola Sicura" program, January-March 2021

\begin{tabular}{|l|r|r|c|}
\hline & \multicolumn{1}{|c|}{ N } & \multicolumn{1}{c|}{$\begin{array}{c}\text { Total in } \\
\text { Piedmont }\end{array}$} & \multicolumn{1}{c|}{$\%$} \\
\hline Participating schools & 428 & 622 & 68.8 \\
\hline Participating classes & 1,942 & 3,856 & 50.4 \\
\hline Students attending participating classes & 41,501 & 78,689 & 52.7 \\
\hline Participating students & 19,413 & 78,689 & 24.7 \\
\hline Students who underwent at least one test & 15,318 & 78,689 & 19.5 \\
\hline - males & 7,891 & 40,910 & 19.3 \\
\hline - females & 7,427 & 37,779 & 19.7 \\
\hline - born in 2007 & 7,250 & 39,069 & 18.6 \\
\hline - born in 2008 & 7,451 & 39,620 & 18.8 \\
\hline
\end{tabular}

There was wide variability in program adherence across the region (Table 2): participation was over $40 \%$ in only two LHUs, while it was less than $10 \%$ in three of them. 
Table 2 - Percentage of students who underwent at least one test, stratified by local health unit (LHU)

\begin{tabular}{|l|r|r|c|}
\hline & $\begin{array}{c}\text { Students who have } \\
\text { undergone at least } \\
\text { one test }\end{array}$ & Total students & $\%$ \\
\hline LHU 1 & 1,645 & 7,196 & 22.9 \\
\hline LHU 2 & 183 & 3,469 & 5.3 \\
\hline LHU 3 & 1,109 & 2,726 & 40.7 \\
\hline LHU 4 & 1,095 & 7,972 & 13.7 \\
\hline LHU 5 & 317 & 3,431 & 9.2 \\
\hline LHU 6 & 3,196 & 6,791 & 47.1 \\
\hline LHU 7 & 2,627 & 15,390 & 17.1 \\
\hline LHU 8 & 1,034 & 10,860 & 9.5 \\
\hline LHU 9 & 1,867 & 9,548 & 19.6 \\
\hline LHU 10 & 822 & 5,955 & 13.8 \\
\hline LHU 11 & 535 & 2,696 & 19.8 \\
\hline LHU 12 & 868 & 2,655 & 32.7 \\
\hline Missing & 20 & & \\
\hline Total & 15,318 & 78,689 & 19.5 \\
\hline
\end{tabular}

The mother-child pair was reconstructed for 12,330 of the 15,318 students who underwent at least one screening swab test. High maternal education was more frequent for those 12,330 students $(20.7 \%)$ than for other Piedmont children of the same age $(14.9 \%, p$ value for the difference < 0.0001 ) who did not contribute to SS.

\section{Results of SS for monitoring purposes (temporal trends and test accuracy)}

The program did not start at the same time in the whole region: testing began in the week 11-17 January 2021; by the week 8-14 February 2021 it had been rolled out in all LHU. As a consequence, 1,000 swab tests were performed on the week 25-31 January 2021 and 3,500 tests were performed in the week 22-28 February 2021.

Out of the 22,128 tests performed during the study period, 114 were positive for SARS-CoV-2. This corresponds to an average proportion of $0.52 \%$ (95\% confidence interval (Cl) 0.42-0.61). However, as shown in Figure 1a, this proportion varied strongly with time. The joint point model showed a significant peaking at week 9 when as much as $1.0 \%(95 \% \mathrm{Cl}: 0.7 \%-1.4 \%)$ of the tested students was positive to SARS-CoV-2.

For the sake of comparison, we report in Figure $1 \mathrm{~b}$ the trend in incidence of positive cases (total $=1,823$ ) identified through tests carried out outside the SS program over the whole population of students of the $2 n d$ and 3 rd grades of first-level secondary schools in Piedmont $(n=78,689)$ during the 12 weeks of the study. The shape of the curve resembles that reported in Figure 1a, although the incidence peaked 1 week later (the joint point model identified a rise at week 6 and a decline beginning after week 10 ).

Each LHU could choose the type of swab test: seven LHUs used only antigen swab tests, three only molecular swab tests, and two used either type. Out of all tests performed within SS, 79\% were antigen swab tests. In 67 cases, an initial positive antigen swab test was followed by a confirmatory molecular test, resulting in 38 confirmed positive and 29 negative results. The mean positive predictive value of the antigen swab tests over the whole study period was therefore $56.7 \%$, implying a $43.3 \%$ probability of a false positive result. The overall proportion of positive cases detected among the tested students was $1.31 \%$ when the molecular swab test was used in the first instance and $0.28 \%$ when the antigen swab test was used in the first instance. 
Figure 1 - Weekly trend of the frequency of positive students within the "Scuola Sicura" program (a) $)^{1}$ and outside the program $(b)^{2}$

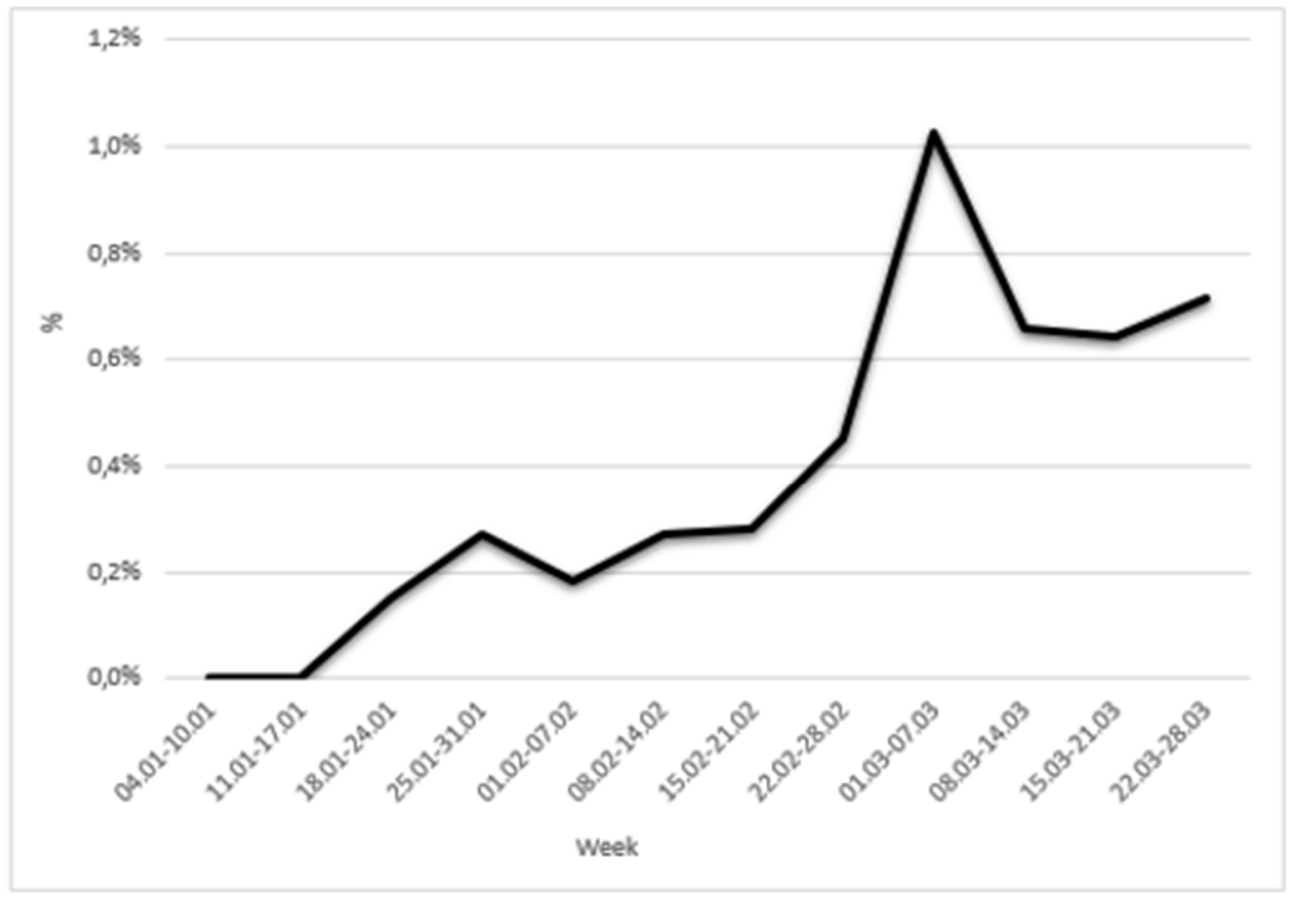

a

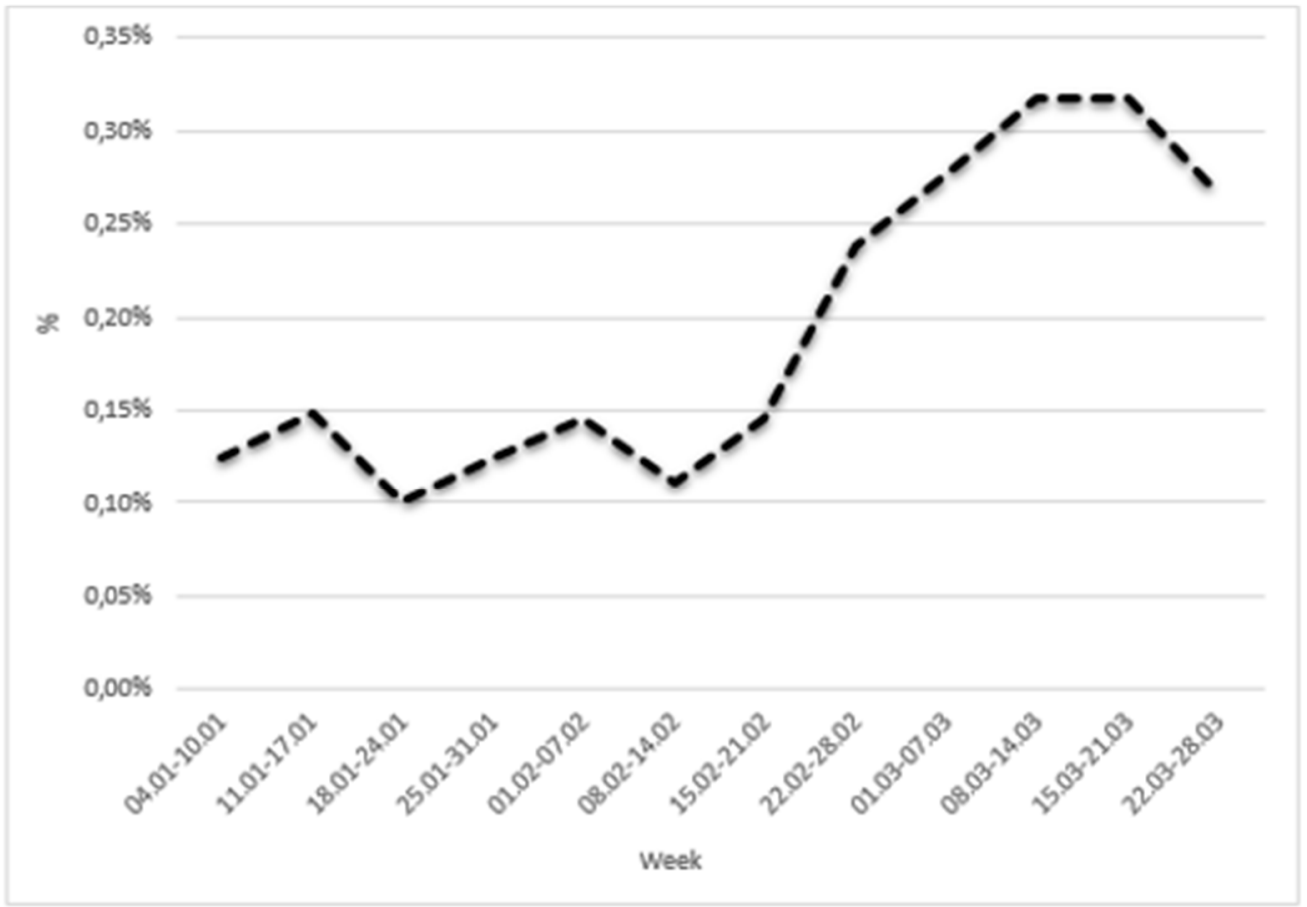

b

${ }^{1}$ The denominator is the number of students tested for screening ( $N$ total in the Region: 22,128 )

2 The denominator is the total number of students in the 2 nd and ard grades of lower secondary schools ( $\mathrm{N}$ total in the Region: 78,689)

9 


\section{Observed preventive capacity of SS}

To estimate the SS preventive capacity, we used LHU data. As reported in Table 3, 69 of the 114 COVID-19 cases discovered within SS were identified before the school closure and the start of remote learning ( 8 March 2021), resulting in the activation of 67 class quarantines. In the same period, in addition to those 69 cases, the standard testing and contact tracing procedures identified 191 other cases among the students of the classes participating in SS, prompting 152 class quarantines. Asymptomatic cases identified at screening accounted for $26.5 \%$ of the total number of cases (69/260).

Table 3 - Positive cases identified in the classes participating in the "Scuola Sicura" program and ouside the program

\begin{tabular}{|c|c|c|c|}
\hline & $\begin{array}{c}\text { Positive cases identified by } \\
\text { the program (\% of the total } \\
\text { positive cases) }\end{array}$ & $\begin{array}{l}\text { Positive cases identified by } \\
\text { contact tracing procedures }\end{array}$ & $\begin{array}{c}\text { Total positive } \\
\text { cases }\end{array}$ \\
\hline \multicolumn{4}{|l|}{ SS $(N=41,501)$} \\
\hline $\begin{array}{l}\text { In-class learning } \\
\text { (January } 7 \text { - March 7) }\end{array}$ & $69(26.5)$ & 191 & 260 \\
\hline $\begin{array}{l}\text { Remote learning } \\
\text { (March 8-28) }\end{array}$ & $45(38.4)$ & 72 & 117 \\
\hline Total & $114(30.2)$ & 263 & 377 \\
\hline \multicolumn{4}{|l|}{ No SS $(\mathrm{N}=37,188)$} \\
\hline $\begin{array}{l}\text { In-class learning } \\
\text { (January } 7-\text { March 7) }\end{array}$ & Not applicable & 922 & 922 \\
\hline $\begin{array}{l}\text { Remote learning } \\
\text { (March 8-28) }\end{array}$ & Not applicable & 638 & 638 \\
\hline Total & Not applicable & 1560 & 1560 \\
\hline
\end{tabular}

Considering that the classes participating in SS are attended by 41,501 students, the 260 cases identified (SS + not SS) over the in-class learning period led to a cumulative incidence of $0.63 \%$ (95\% $\mathrm{Cl}: 0.55 \%-0.71 \%)$.

As reported in Table 3, during the same period, the standard testing and contact tracing procedures identified 922 cases among the 37,188 students belonging to classes that did not participate in SS, corresponding to a cumulative incidence of $2.48 \%$ (95\% Cl: $2.32 \%-2.64 \%)$.

The mother-child pair was identified in 61 of the 69 cases (88\%) detected through the screening before 8 March 2021; 46 (75\%) mothers had undergone antigen or molecular swab testing in the 21 days after the test date of their child, and 11 mothers (18\%) resulted positive for SARS-CoV-2.

\section{Discussion}

During the first quarter of 2021 a region-wide screening study involved students attending second and third grades of first-level secondary schools in Piedmont. Screening was offered in addition to other mitigation strategies against the spread of COVID-19 in schools. The organization of screening was based on simulations and designed to maximize efficiency and outcome ${ }^{14}$ and to reduce the number of rhino-pharyngeal swab tests that each child could undergo. This is in line with the recommendations by the U.S. Centers for Disease Control that school screening may be useful in containing spread of the virus when community transmission is high or moderate, when offered 
frequently, when the test results are obtained rapidly, and measures for response to identification of positive cases are clearly defined. ${ }^{15}$

\section{Program adherence}

Although $70 \%$ of the schools in Piedmont participated in the program, student adherence, which was on a voluntary basis, was $19.5 \%$ of the total target population, lower than expected. While there were no differences by age and sex, adherence rates differed widely by geographical area of the region. This finding cannot be compared with previous studies in which adherence was obligatory for all students of schools participating in similar programs. ${ }^{9,10}$

The high geographic variability suggests that barriers to participation may be linked to the local organizational context and the active role of schools in stimulating adherence and participation. The imbalance towards the high level of education of the mother of the students who underwent at least one test further highlights the importance of conveying correct messages during the enrolment phase.

We have discussed these results together with the LHU health workers. One possible explanation for the low adherence could be related to communication issues, such as difficulties of schools and health offices in clearly and timely informing students' families about the program objectives, identifying the criticalities, and devising ways to resolve them. The program began when the schools reopened after the Christmas break; the students returned to class after a period of remote learning that had begun in November 2020 and coincided with the second wave of the pandemic. It is possible that families that had troubles in managing family life during the previous months of the pandemic were worried about the organizational and financial consequences of continued remote learning if their child or their classmates tested positive at screening and were quarantined. The offices of the school principals, which were responsible for liaising with the families, worked differently in their districts, reaching different levels of communicative efficacy, also based on the collaborative relationship between schools and LHUs. Communication with the students' families, which is a key component of voluntary screening, needs to be revised and strengthened if the program is repeated, also to counteract the imbalance towards the high educational levels of the participant mothers.

Another potential reason for the low adherence was the difficulty of families to arrange for their children to come to the test centers. In other countries, screening was performed at the school $\left.\right|^{9,10}$ or by means of at-home self-test kits. ${ }^{16}$ Before the program can be repeated, the feasibility of such methods needs to be evaluated.

\section{Weekly trends}

We compared the weekly trends of the frequency of positive students within SS and outside the program, in the hypothesis that SS could anticipate epidemic peaks (as it usually happens in a screening program). The comparison of weekly trends showed indeed that the slope of the curve began to decline one week earlier in March for the students participating than for those not participating in the program; this was not observed, as expected, for the rising slope of the curve. A plausible explanation for the difference is that very few cases were detected among the students participating in the program in the first weeks, resulting in very low incidence and almost no weekly change. More robust data might have revealed an earlier upward slope of the curve.

\section{Test accuracy}

The LHUs were free to choose the type of swab test and were responsible for ensuring test accuracy (higher for molecular tests) and obtaining prompt results (faster for antigen tests). The positive predictive value of the antigen tests (56.7\%), which is quite low, may have caused inconvenience to 
students and their families (false positive result of the antigen test would have meant that the entire class remained at home and attended school remotely until the molecular test resulted negative for the virus). Furthermore, as supported by the results of this work, molecular swab tests can identify a greater number of asymptomatic children with COVID-19, because they have a higher sensitivity and consequently, they reduce the impact of false negatives on the efficacy of the screening. Our study findings support the importance of using tests with a high specificity and sensitivity. When antigen swab tests are used, tests of third generation should be chosen.

\section{Preventive capacity}

The third wave of the pandemic struck Italy during the study period. The community incidence of new cases in Piedmont was 161 per 100,000 (6,989 cases, Rt. 1.09) in the week 4-10 January 2021 and 344 (14,830 cases, Rt 1.16) in the week 15-21 March 2021, when the wave peaked. The Minister of health issued a nationwide order for remote learning that included second and third graders in first-level secondary schools, starting on 8 March 2021. Evaluation of the preventive capacity of the program was limited to the period of in-class learning (7 January to 7 March 2021). Screening led to the detection of $26.5 \%$ of the positive cases identified in the adherent classes, meaning that contact tracing procedures had lost a substantial percentage of cases. This observation is shared by Denny et $a l^{12}$ in their study on screening at Duke University (testing at the start of the semester and then twice weekly), by which they identified $55 \%$ of all cases reported during the study period. If all the students in the target population had participated in the program, implying that a quarter of the students were tested weekly for 9 weeks, then about 450 additional cases might have been detected. These results underline the importance of coupling school screening with contact tracing.

Nearly all cases detected by screening led to quarantine for the entire class (67/69), stimulating testing also in family members and therefore identifying other asymptomatic cases. The procedures carried out following the detection of a positive case at screening seem to have been more efficient than quarantining of positive cases detected outside the program ( $97.1 \%$ vs. $79.6 \%)$. Moreover, the frequency of positive cases in the participating classes was much lower than the same frequency in the non participating classes ( 0.63 vs. $2.48 \%$ ). It should be emphasized that adherence of the classes to the screening was not randomized. The low frequency observed in the classes adhering to the program from one hand could be due to a higher students' participation in areas with low levels of virus circulation, or a higher compliance with the other mitigation measures, or a lower risk of infection outside schools; on the other hand, it could suggest that screening plus the other mitigation measures may have helped keep virus circulation low in the participating classes.

\section{Strengths and limitations}

This is one of the few studies (and the first in Italy) to describe the functioning and the observed predictive capacity of school screening testing for SARS-CoV-2 in a real-world situation. Despite considerable geographic and social variability, the program involved thousands of school students throughout the region, unlike most previous studies which reported on programs conducted at single schools.

We were unable to estimate the total observed preventive capacity of screening in the school setting, because the Ministry of health protocols do not include testing of the classmates of students found positive for the virus. We can estimate, however, that another 50-60 asymptomatic students in the quarantined classes were positive, based on the mean secondary attack rate $(3.8 \%)$ reported in a study conducted in Reggio Emilia during the second wave of the pandemic ${ }^{17}$ and given an average class size of $20-23$ students. 
Moreover, we were unable to estimate the preventive capacity for the households. Though the ministerial procedure foresees quarantining and testing of household members of positive students, the regional data platform does not enable the complete reconstruction of households nor of the exact dynamics of household transmission. Nonetheless, we know that at least 11 mothers of students who tested positive during in-class lessons (out of 61 identified pairs) tested positive within 21 days after the notification of their child's test results.

\section{Conclusions}

Our findings provide many operative suggestions. Schools and local health units should work together, in an integrated way, and build groups of dedicated and specifically trained personnel. A wide communication plan must be defined, including strategies to stimulate the participation of disadvantaged families. Molecular tests or antigenic tests of third generation should be used, and the feasibility of administering them directly at schools should be evaluated. A dedicated information system must be built and results must be monitored. When well organized and implemented, school screening can make an important contribution in identifying and isolating asymptomatic cases. Jointly with contact tracing and other measures of transmission prevention, it may be a viable strategy to keep schools open when high levels of the virus are circulating in the community.

Further analysis of data is planned for a period of low prevalence of COVID-19 in the general population (April-June 2021). Though over time a greater number of teachers and students will probably have been vaccinated, some members of the school community may not. Furthermore, we know little about the risk of asymptomatic transmission among the vaccinated, particularly for new variants. ${ }^{18}$ Although in a new scenario, strategies different from that used in the present study could be adopted (e.g. saliva tests or pooling strategies), our findings provide data-driven suggestions for government agencies when planning large-scale screening school programs.

Conflict of interest: none declared.

\section{Acknowledgements:}

This paper was based on the work of the schools and the covid-school groups of the Local Health Units of the Piedmont.

The authors would like to thank Francesco Tresso and Giorgio Nocerino of CSI Piemonte for the support and collaboration. 


\section{Bibliografia}

1. Thompson HA, Mousa A, Dighe A, et al. SARS-CoV-2 setting-specific transmission rates: a systematic review and meta-analysis. Clin Infect Dis 2021:ciab100:1-34. doi: 10.1093/cid/ciab100 pmid: 33560412

2. Viner RM, Mytton OT, Bonell C, et al. Susceptibility to SARS-CoV-2 infection among children and adolescents compared with adults: a systematic review and meta-analysis. JAMA Pediatr 2021;175:143-56. doi: 10.1001/jamapediatrics.2020.4573 pmid: 32975552

3. Lewis SJ, Munro APS, Smith GD, Pollock AM. Closing schools is not evidence based and harms children. Keeping schools open should be the UK's top priority. BMJ 2021;372:n521:1-2. http://dx.doi.org/10.1136/bmj.n521

4. Viner RM, Russell S, Saulle R, et al. Impacts of school closures on physical and mental health of children and young people: a systematic review. medRxiv 2021.02.10.21251526; doi: https://doi.org/10.1101/2021.02.10.21251526

5. Christakis DA. Estimation of US Children's Educational Attainment and Years of Life Lost Associated With Primary School Closures During the Coronavirus Disease 2019 Pandemic. JAMA Netw Open. 2020 Nov 2;3(11):e2028786:1-12. doi: 10.1001/jamanetworkopen.2020.28786

6. European Centre for Disease Prevention and Control. COVID-19 in children and the role of school settings in transmission-first update. 2020:1-57. https://www.ecdc.europa.eu/en/publicationsdata/children-and-school-settings-covid-19-transmission

7. World Health Organization, Unicef, Unesco. Considerations for school-related public health measures in the context of COVID-19: annex to considerations in adjusting public health and social measures in the context of COVID-19. 2020. https://apps.who.int/iris/handle/10665/334294

8. Rafiei Y, Mello MM. The Missing Piece - SARS-CoV-2 Testing and School Reopening. N EngI J Med 2020; 383:e126 DOI: 10.1056/NEJMp2028209

9. Volpp KG, Kraut BH, Ghosh S, Neatherlin J. Minimal SARS-CoV-2 Transmission After Implementation of a Comprehensive Mitigation Strategy at a School - New Jersey, August 20November 27, 2020. MMWR / March 19, 2021 / Vol. 70 / No. 11 /377-81

10. Berke EM, Newman LM, Jemsby S, et al. Pooling-in-a-pod: A Strategy for COVID-19 Testing to Facilitate Safe Return to School. Preprint. DOI: https://doi.org/10.1101/2021.03.24.21254230

11. Kriemler S, Ulyte A, Ammann P, et al. Surveillance of Acute SARS-CoV-2 Infections in School Children and Point-Prevalence During a Time of High Community Transmission in Switzerland. Front. Pediatr., 16 March 2021 /1-8 | https://doi.org/10.3389/fped.2021.645577.

12. Denny TN, Andrews L, Bonsignori M, et al. Implementation of a Pooled Surveillance Testing Program for Asymptomatic SARS-CoV-2 Infections on a College Campus - Duke University, Durham, North Carolina, August 2-October 11. MMWR / November 20, 2020 / Vol. 69 / No. 46 / 1743-47

13. WHO Regional Office for Europe. Schooling during COVID-19: recommendations from the European Technical Advisory Group for schooling during COVID-19. Copenhagen, 2021. Licence: CC BY-NC-SA 3.0 IGO.

14. Baccini M, Cereda G. Screening plans for SARS-CoV-2 based on sampling and rotation: an example in the school setting. Preprint. DOI: https://doi.org/10.1101/2021.02.10.21251502

15. Centers for desease control and prevention. Operational Strategy for K-12 Schools through Phased Prevention. Updates as of May 15, 2021, 1-24. https://www.cdc.gov/coronavirus/2019ncov/community/schools-childcare/operation-strategy.html

16. National Health System. Regular rapid lateral flow coronavirus (COVID-19) tests. Page last reviewed: 29 June 2021. Regular rapid lateral flow coronavirus (COVID-19) tests - NHS (www.nhs.uk) 17. Larosa E, Djuric O, Cassinadri M, et al, the Reggio Emilia COVID-19 Working Group. Secondary transmission of COVID-19 in preschool and school settings in northern Italy after their reopening in September 2020: a population-based study. 1-13. www.eurosurveillance.org 
18. Public Health England. SARS-CoV-2 variants of concern and variants under investigation in England. Technical briefing 16. 18 June 2021 -1-71. 